\title{
ASSESSMENT OBESITY AMONG CHILDREN IN LIKONI SUBCOUNTY, MOMBASA COUNTY
}

\author{
Kailong J. M. ${ }^{1}$ \\ ${ }^{1}$ Assistant lecturer, \\ Department of medical sciences, \\ School of pure and health sciences, \\ Technical University of Mombasa, \\ Kenya.
}

\author{
Adem A, \\ ${ }^{2}$ Lecturer, \\ Department of mathematics and Physics, \\ School of pure and health sciences, \\ Technical University of Mombasa, \\ Kenya.
}

Article DOI: https://doi.org/10.36713/epra5836

\begin{abstract}
Obesity is now becoming a global problem, causing many life threatening lifestyle diseases. The burden is even more worrying among the young children in the developing countries like Kenya because few studies have been done. The purpose of the study was undertake an assessment of Obesity prevalence and precipitating factors among lower primary school children in Likoni Sub County, Mombasa County, Kenya. The study employed a descriptive cross sectional study and the school children of between grade 1-4 in both private and public schools being the target population. Sample sizes of 384 participants were recruited using Cochrans (2010) formula. Structured questionnaire was used to collect quantitative data, observational checklist and anthropometric measurements were used to collect qualitative data. Data was analyzed using SPSS version 20 and interpreted using tables and pie charts. The study findings show that obesity stands at 23\% among respondents. According to BMI interpretations there were $2 \%$ under weight, $69.1 \%$ normal in public schools, while over weight (28.1) and obesity (32.6\%) was high in private school children. There was a significant association between means of transport $(p=0.000)$, leisure time $(p=0.000)$, Grade $(0.000)$, residence $(0.000)$ occupation $(0.000)$ and income (p=0.000). The determinants to obesity in children were residence (AOR 29.46; CI 10.054, 86.34) and between break meals (AOR 4.647; CI 1.958,11.025).
\end{abstract}

\section{INTRODUCTION}

About 1 billion people were classified overweight and 300 million being obese (WHO, 2002) Globally, nearly 1 billion people are classified as overweight, 300 million of them being clinically obese (WHO, 2002). Nearly one third of the adult American population is obese, while in South Africa, more than one in two adult women are overweight or obese. In Morocco, $40 \%$ of the population is overweight (Bertone, Staneck and Cohen, 2003).

Overweight and obesity in developing countries, has been neglected as most attention is concentrated on famine and under nutrition or malnutrition of children (Malla, 2004 and Kamau 2008). If preventive measures are not put in place, the problem may escalate and overburden the health care system in these areas (Caroli and Burniat, 2002). Hence there is need to put measures in place to arrest the problem of overweight and obesity and prevent the negative consequences

\section{OBJECTIVES}

\section{General objectives}

Assessment of obesity among lower primary school children in Likoni sub county, Mombasa County

Specific objectives

a) To determine the proportion of obesity among children in primary schools 
b) To establish the primary school children learning environment in relation to obesity

c) To determine parents influence on obesity in primary school children

\section{METHODOLOGY}

This study descriptive cross-sectional to with aim to collect both qualitative and quantitative data related to overweight and obesity among school going children in public and private schools in Likoni sub county, Mombasa County

The design was appropriate in the study of prevalence of overweight and obesity among children. The study was useful in determining the associated factors to overweight and obesity and how to minimize them

\section{Sampling}

The study was carried out among ten primary schools ( 5 private and 5 public) all found in Likoni. The target population was Grade 1, 2, $3 \& 4$ children as well as their parents. Systematic random sampling was used to recruit the children in the specific school at an interval of five until required sample size was realized. Every recruited child was given a questionnaire and a consent form to take to his/her parent. The parent could go through the questions at home and voluntary sign the consent and fill the questionnaires. The next day, all returned questionnaires by children and consent form were collected and particular child with the help of the teacher taken through anthropometric measurements. The recruitment continued in every school until desired sample size achieved. All subjects were taken through the study objectives, benefits and risks before consenting. Confidentiality was assured at every stage of this study

The collected data was edited then entered into SPSS software (version 21) for analysis based on the study variables. The study results was presented using tables and pie charts

\section{Sample size}

The study sample size was calculated using the Cochran's formula (Cochran's, 2010)

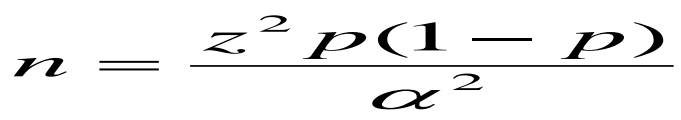

Where:

$\mathrm{n}=$ Minimum required sample size

$\mathrm{z}=$ Reliability coefficient (1.96 at $95 \%$

confidence interval)

$\mathrm{p}=$ Estimated proportion of PLHIV who have disclosed their HIV status taken to be $49 \%$.

$\alpha=$ Maximum likely error (5\%)

Therefore, the minimum sample size will be given as;

$$
n=\frac{1.96^{2} \times 0.49 \times(1-0.49)}{0.05^{2}}=384
$$

\section{Study Area}

The study was undertaken among primary schools in Likoni Sub County, Likoni constituency, Mombasa County. Likoni Sub County is the main land after crossing the ferry from Mombasa town. It borders Kwale County in the north and Indian Ocean to the south the main economic activities that are carried in this region are fishing, tourism and business at the busiest Likoni ferry which is the largest in Kenya (MOE, 2019). 


\section{RESULTS}

Figures

\section{Obesity}

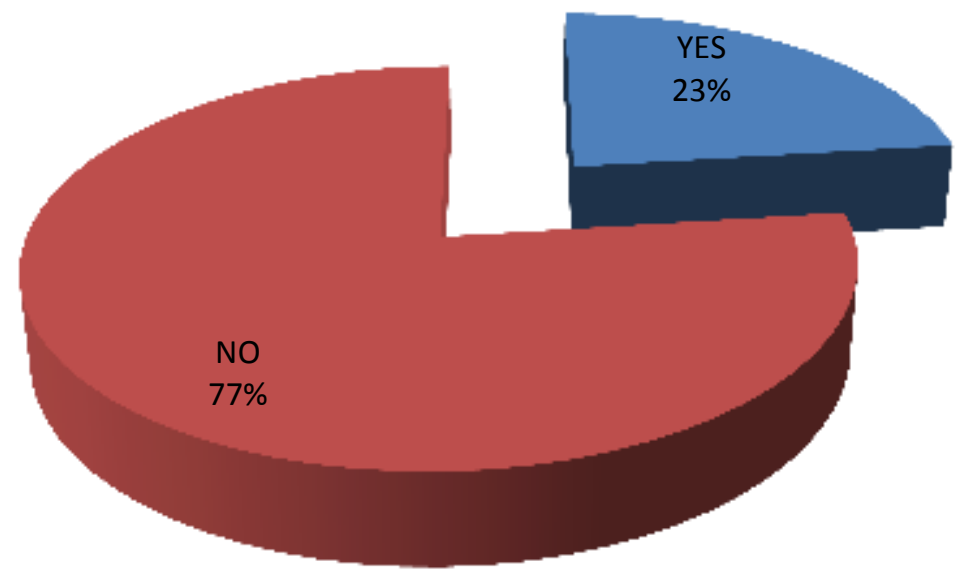

Figure 1: Extent of obesity among the respondents

Tables

Table 1: Socio demographic factors of the respondents

\begin{tabular}{|l|c|c|c|c|}
\hline Variable & Category & Frequency \% & \multicolumn{2}{|c|}{ Schools } \\
\cline { 3 - 5 } & & & Public (n=107) & Private (n=89) \\
\hline Children & & & & \\
\hline Gender & Male & $101(51.5)$ & $52(48.6)$ & $49(55.1)$ \\
\hline \multirow{4}{*}{ Daily Pocket money (Ksh.) } & Female & $95(48.5)$ & $55(51.4)$ & $40(44.9)$ \\
\cline { 2 - 5 } & $0-100$ & $18(9.2)$ & $11(10.3)$ & $7(7.8)$ \\
\cline { 2 - 5 } & $100-200$ & $107(54.6)$ & $53(49.5)$ & $54(60.7)$ \\
\cline { 2 - 5 } & $>200$ & $71(36.2)$ & $43(40.2)$ & $28(31.5)$ \\
\hline \multirow{4}{*}{ Means of transport } & School van & $135(68.9)$ & $65(60.7)$ & $70(78.7)$ \\
\cline { 2 - 5 } & Walking & $24(12.2)$ & $22(20.6)$ & $2(2.2)$ \\
\cline { 2 - 5 } & Others & $37(18.9)$ & $20(18.7)$ & $17(19.1)$ \\
\cline { 2 - 5 } & Video games & $152(77.6)$ & $70(65.4)$ & $82(92.2)$ \\
\cline { 2 - 5 } & Watch TV & $14(7.1)$ & $12(11.2)$ & $2(2.2)$ \\
\cline { 2 - 5 } & Playing sports & $8(4.1)$ & $5(4.7)$ & $2(3.4)$ \\
\hline Grade & Sleeping & $22(11.2)$ & $18(17.1)$ & $22(24.7)$ \\
\cline { 2 - 5 } & 1 & $40(20.4)$ & $29(11.2)$ & $20(22.5)$ \\
\cline { 2 - 5 } & 2 & $32(16.3)$ & $48(44.6)$ & $29(32.6)$ \\
\cline { 2 - 5 } & 3 & $58(29.6)$ & $61(57)$ & $18(20.2)$ \\
\hline Parents & 4 & $66(33.7)$ & & $4(4.5)$ \\
\hline Residence & Rental house & $65(33.2)$ & & \\
\hline
\end{tabular}


ISSN (Online): 2455-3662

EPRA International Journal of Multidisciplinary Research (IJMR) - Peer Reviewed Journal

Volume: 7 | Issue: 1 |January 2021|| Journal DOI: 10.36713/epra2013 || SJIF Impact Factor: 7.032 ||ISI Value: 1.188

\begin{tabular}{|l|c|c|c|c|}
\hline & Personal house & $131(66.8)$ & $46(43)$ & $85(95.5)$ \\
\hline \multirow{3}{*}{ Occupation } & Employed & $145(74)$ & $59(55.1)$ & $86(96.6)$ \\
\cline { 2 - 4 } & Unemployed & $51(26)$ & $48(44.9)$ & $3(3.4)$ \\
\hline \multirow{3}{*}{ Income (Ksh.) } & $<10,000$ & $2(1)$ & $2(1.9)$ & $0(0)$ \\
\cline { 2 - 4 } & $10,000-20,000$ & $42(21.4)$ & $36(33.6)$ & $6(6.7)$ \\
\cline { 2 - 4 } & $>20,000$ & $152(77.6)$ & $69(64.5)$ & $83(93.3)$ \\
\cline { 2 - 4 } & Married & $158(80.6)$ & $75(70.1)$ & $75(84.3)$ \\
\cline { 2 - 4 } & Single / divorced & $38(19.4)$ & $24(29.9)$ & $14(15.7)$ \\
\hline
\end{tabular}

Table 2: School environment

\begin{tabular}{|c|c|c|c|c|}
\hline \multirow[t]{2}{*}{ Variable } & \multirow[t]{2}{*}{ Category } & \multirow[t]{2}{*}{ Frequency $(\mathrm{N}=196)$} & \multicolumn{2}{|c|}{ Schools } \\
\hline & & & Public (n=107) & Private $(n=89)$ \\
\hline \multirow[t]{3}{*}{ Physical activities } & Sports equipments & $89(45.4 \%)$ & $29(27.1 \%)$ & $60(67.4 \%)$ \\
\hline & Sport lessons & $45(23 \%)$ & $42(39.3 \%)$ & $3(3.8 \%)$ \\
\hline & Sport activities & $62(31.6 \%)$ & $36(33.6 \%)$ & $26(29.2 \%)$ \\
\hline \multirow[t]{4}{*}{ Eating habits } & Food shops & $33(16.8 \%)$ & $20(18.7 \%)$ & $13(14.6 \%)$ \\
\hline & Food from outside & $45(23 \%)$ & $2(1.95)$ & $43(48.3 \%)$ \\
\hline & Food hawkers & $32(16.3 \%)$ & $24(22.4 \%)$ & $8(9 \%)$ \\
\hline & Food served in school & $86(43.9 \%)$ & $61(57 \%)$ & $25(28.1 \%)$ \\
\hline \multirow[t]{2}{*}{ School location } & Residential area & $124(63.3 \%)$ & $53(49.5 \%)$ & $71(79.8 \%)$ \\
\hline & Has play ground & $72(36.7 \%)$ & $54(50.5 \%)$ & $18(20.2 \%)$ \\
\hline \multicolumn{5}{|l|}{ Food served } \\
\hline \multirow[t]{3}{*}{ Main meal } & Rice+beans & $64(32.7 \%)$ & $42(39.3 \%)$ & $22(24.7 \%)$ \\
\hline & Beans+ chapati & $94(48 \%)$ & $37(34.6 \%)$ & $57(64 \%)$ \\
\hline & Ugali+vegetables & $38(19.3 \%)$ & $28(26.2 \%)$ & $10(11.2 \%)$ \\
\hline \multirow{2}{*}{$\begin{array}{ll}\text { Between } & \text { Break } \\
\text { meals } & \end{array}$} & Tea + mahamri & $94(48 \%)$ & $82(76.6 \%)$ & $12(13.5 \%)$ \\
\hline & Tea + cake & $102(52 \%)$ & $25(23.4 \%)$ & $77(86.5 \%)$ \\
\hline \multirow[t]{3}{*}{ Servings } & Once & $97(49.5 \%)$ & $94(87.9 \%)$ & $3(3.4 \%)$ \\
\hline & Twice & $77(39.3 \%)$ & $13(12.1 \%)$ & $64(71.9 \%)$ \\
\hline & $>$ twice & $22(11.2 \%)$ & $0(0)$ & $22(24.7 \%)$ \\
\hline \multirow[t]{7}{*}{ Snacks } & Chocolate & $56(28.5 \%)$ & $27(25.2 \%)$ & $29(32.6 \%)$ \\
\hline & Chips & $43(22 \%)$ & $19(17.8 \%)$ & $24(27 \%)$ \\
\hline & Juice & $36(18.4 \%)$ & $27(25.2 \%)$ & $9(10.1 \%)$ \\
\hline & Biscuits & $14(7.1 \%)$ & $4(3.7 \%)$ & $10(11.2 \%)$ \\
\hline & Crisps & $13(6.6 \%)$ & $6(5.6 \%)$ & $7(7.9 \%)$ \\
\hline & Ice cream & $25(12.8 \%)$ & $17(15.9 \%)$ & $8(9 \%)$ \\
\hline & Sweets & $9(4.6 \%)$ & $7(6.5 \%)$ & $2(2.2 \%)$ \\
\hline
\end{tabular}

Table 3: Anthropometric measures

\begin{tabular}{|c|c|c|c|}
\hline BMI & \multicolumn{2}{|c|}{ School } & Frequency \\
\hline & Public & Private & Total \\
\hline Underweight & $2(1.9)$ & $2(2.2)$ & $4(2 \%)$ \\
\hline Normal & $74(69.1)$ & $33(37.1)$ & $107(54.6 \%)$ \\
\hline Over weight & $15(14)$ & $25(28.1)$ & $40(20.4 \%)$ \\
\hline Obese & $16(15)$ & $29(32.6)$ & $45(23 \%)$ \\
\hline
\end{tabular}

Table 4: Schools anthropometric measurements

\begin{tabular}{|c|c|c|c|c|}
\hline Public & Underweight & Normal & Overweight & Obesity \\
\hline Mrima primary school & $1(25 \%)$ & $18(16.85)$ & $2(5 \%)$ & $2(4.4 \%)$ \\
\hline $\begin{array}{c}\text { Likoni muslim primary } \\
\text { school }\end{array}$ & 0 & $22(20.6 \%)$ & $3(7.5 \%)$ & $2(4.4 \%)$ \\
\hline
\end{tabular}


ISSN (Online): 2455-3662

EPRA International Journal of Multidisciplinary Research (IJMR) - Peer Reviewed Journal

Volume: 7 | Issue: 1 |January 2021|| Journal DOI: 10.36713/epra2013 || SJIF Impact Factor: 7.032 ||ISI Value: 1.188

\begin{tabular}{|c|c|c|c|c|}
\hline Likoni primary school & $1(25 \%)$ & $9(8.4 \%)$ & $2(5 \%)$ & $3(6.8 \%)$ \\
\hline Puma primary school & 0 & $14(13.1 \%)$ & $4(10 \%)$ & $5(11.1 \%)$ \\
\hline Maji safi primary school & 0 & $11(10.3 \%)$ & $4(10 \%)$ & $4(8.9 \%)$ \\
\hline Private & 0 & & & \\
\hline Alnoor integrated school & 0 & $7(6.55 \%)$ & $8(20 \%)$ & $10(22.2 \%)$ \\
\hline Kenya excellent academy & $1(25 \%)$ & $3(2.8 \%)$ & $3(7.5 \%)$ & $2(4.4 \%)$ \\
\hline $\begin{array}{c}\text { Al Miraj integrated } \\
\text { academy }\end{array}$ & 0 & $6(5.6 \%)$ & $6(15 \%)$ & $8(17.8 \%)$ \\
\hline Oceania academy & 0 & $8(7.5 \%)$ & $4(10 \%)$ & $5(11.1 \%)$ \\
\hline Sitra integrated academy & $1(25 \%)$ & $9(8.4 \%)$ & $4(10 \%)$ & $4(8.9 \%)$ \\
\hline Total & $2(100 \%)$ & $107(100 \%)$ & $40(100 \%)$ & $45(100 \%)$ \\
\hline
\end{tabular}

Table 5: Bivariate analysis on socio demographic factors in relation to Obesity

\begin{tabular}{|c|c|c|c|c|c|c|c|c|}
\hline \multirow[t]{2}{*}{ Variable } & \multirow[t]{2}{*}{ Category } & \multirow{2}{*}{$\begin{array}{l}\text { Frequency } \\
\%(\mathrm{~N}=196)\end{array}$} & \multicolumn{2}{|c|}{ Schools } & \multirow{2}{*}{ Df } & \multirow{2}{*}{$\begin{array}{c}\text { Chi } \\
\text { square }\end{array}$} & (2) & \multirow[b]{2}{*}{ OR } \\
\hline & & & $\begin{array}{c}\text { Public } \\
(n=107)\end{array}$ & $\begin{array}{l}\text { Private } \\
(n=89)\end{array}$ & & & P value & \\
\hline \multicolumn{9}{|l|}{ Children } \\
\hline \multirow[t]{2}{*}{ Gender } & Male & $101(51.5)$ & $52(48.6)$ & $49(55.1)$ & \multirow[t]{2}{*}{1} & \multirow[t]{2}{*}{0.811} & \multirow[t]{2}{*}{0.368} & \multirow[t]{2}{*}{$0.772,(0.439,1.357)$} \\
\hline & Female & $95(48.5)$ & $55(51.4)$ & $40(44.9)$ & & & & \\
\hline \multirow{3}{*}{$\begin{array}{c}\text { Daily Pocket } \\
\text { money } \\
\text { (Ksh.) }\end{array}$} & $0-100$ & $18(9.2)$ & $11(10.3)$ & $7(7.8)$ & \multirow[t]{3}{*}{2} & \multirow[t]{3}{*}{2.435} & \multirow[t]{3}{*}{0.296} & \multirow[t]{3}{*}{-} \\
\hline & $100-200$ & $107(54.6)$ & $53(49.5)$ & $54(60.7)$ & & & & \\
\hline & $>200$ & $71(36.2)$ & $43(40.2)$ & $28(31.5)$ & & & & \\
\hline \multirow{3}{*}{$\begin{array}{l}\text { Means of } \\
\text { transport }\end{array}$} & School van & $135(68.9)$ & $65(60.7)$ & $70(78.7)$ & \multirow[t]{3}{*}{2} & \multirow[t]{3}{*}{15.573} & \multirow[t]{3}{*}{0.000} & \multirow[t]{3}{*}{-} \\
\hline & Walking & $24(12.2)$ & $22(20.6)$ & $2(2.2)$ & & & & \\
\hline & Others & $37(18.9)$ & $20(18.7)$ & $17(19.1)$ & & & & \\
\hline \multirow[t]{4}{*}{ Leisure time } & Video games & $152(77.6)$ & $70(65.4)$ & $82(92.2)$ & \multirow[t]{4}{*}{3} & \multirow[t]{4}{*}{28.480} & \multirow[t]{4}{*}{0.000} & \multirow[t]{4}{*}{-} \\
\hline & Watch TV & $14(7.1)$ & $12(11.2)$ & $2(2.2)$ & & & & \\
\hline & Playing sports & $8(4.1)$ & $5(4.7)$ & $3(3.4)$ & & & & \\
\hline & Sleeping & $22(11.2)$ & $20(18.7)$ & $2(2.2)$ & & & & \\
\hline \multirow[t]{4}{*}{ Grade } & 1 & $40(20.4)$ & $18(17.1)$ & $22(24.7)$ & 3 & 57.919 & 0.000 & \\
\hline & 2 & $32(16.3)$ & $12(11.2)$ & $20(22.5)$ & & & & \\
\hline & 3 & $58(29.6)$ & $29(27.1)$ & $29(32.6)$ & & & & \\
\hline & 4 & $66(33.7)$ & $48(44.6)$ & $18(20.2)$ & & & & \\
\hline Parents & & & & & & & & \\
\hline Residence & Rental house & $65(33.2)$ & $61(57)$ & $4(4.5)$ & 1 & 62.119 & 0.000 & 29.46, (10.054, \\
\hline & $\begin{array}{l}\text { Personal } \\
\text { house }\end{array}$ & $131(66.8)$ & $46(43)$ & $85(95.5)$ & & & & 86.34) \\
\hline Occupation & Employed & $145(74)$ & $59(55.1)$ & $86(96.6)$ & 1 & 43.447 & 0.000 & $0.043,(0.013,0.144)$ \\
\hline & Unemployed & $51(26)$ & $48(44.9)$ & $3(3.4)$ & & & & \\
\hline Income & $<10,000$ & $2(1)$ & $2(1.9)$ & $0(0)$ & 2 & 38.632 & 0.000 & - \\
\hline (Ksh.) & $\begin{array}{c}10,000- \\
20,000\end{array}$ & $42(21.4)$ & $36(33.6)$ & $6(6.7)$ & & & & \\
\hline & $>20,000$ & $152(77.6)$ & $69(64.5)$ & $83(93.3)$ & & & & \\
\hline Marital & Married & $158(80.6)$ & $75(70.1)$ & $75(84.3)$ & 1 & 2.106 & 0.147 & $0.583,(0.280,1.218)$ \\
\hline status & $\begin{array}{l}\text { Single / } \\
\text { divorced }\end{array}$ & $38(19.4)$ & $24(29.9)$ & $14(15.7)$ & & & & \\
\hline
\end{tabular}


Table 6: School environment

\begin{tabular}{|c|c|c|c|c|c|c|c|c|}
\hline \multirow{2}{*}{$\begin{array}{c}\text { Variabl } \\
\text { e }\end{array}$} & \multirow[t]{2}{*}{ Category } & \multirow{2}{*}{$\begin{array}{c}\text { Frequency } \\
(N=196)\end{array}$} & \multicolumn{2}{|c|}{ Schools } & \multirow[b]{2}{*}{ Df } & \multirow[b]{2}{*}{$\begin{array}{c}\text { Chi } \\
\text { square }\end{array}$} & \multirow[b]{2}{*}{ P value } & \multirow[b]{2}{*}{ OR } \\
\hline & & & $\begin{array}{c}\text { Public } \\
(\mathrm{n}=107)\end{array}$ & $\begin{array}{l}\text { Private } \\
(\mathrm{n}=89)\end{array}$ & & & & \\
\hline \multirow{3}{*}{$\begin{array}{l}\text { Physical } \\
\text { activities }\end{array}$} & Sports equipments & $89(45.4 \%)$ & $29(27.1 \%)$ & $60(67.4 \%)$ & \multirow[t]{3}{*}{2} & \multirow[t]{3}{*}{44.937} & \multirow[t]{3}{*}{0.000} & \\
\hline & Sport lessons & $45(23 \%)$ & $42(39.3 \%)$ & $3(3.8 \%)$ & & & & \\
\hline & Sport activities & $62(31.6 \%)$ & $36(33.6 \%)$ & $26(29.2 \%)$ & & & & \\
\hline \multirow{4}{*}{$\begin{array}{l}\text { Eating } \\
\text { habits }\end{array}$} & Food shops & $33(16.8 \%)$ & $20(18.7 \%)$ & $13(14.6 \%)$ & \multirow[t]{4}{*}{3} & \multirow[t]{4}{*}{124.198} & \multirow[t]{4}{*}{0.000} & \\
\hline & Food from outside & $45(23 \%)$ & $2(1.95)$ & $43(48.3 \%)$ & & & & \\
\hline & Food hawkers & $32(16.3 \%)$ & $24(22.4 \%)$ & $8(9 \%)$ & & & & \\
\hline & Food served in school & $86(43.9 \%)$ & $61(57 \%)$ & $25(28.1 \%)$ & & & & \\
\hline \multirow{2}{*}{$\begin{array}{c}\text { School } \\
\text { location }\end{array}$} & Residential area & $124(63.3 \%)$ & $53(49.5 \%)$ & $71(79.8 \%)$ & \multirow[t]{2}{*}{1} & \multirow[t]{2}{*}{19.121} & \multirow[t]{2}{*}{0.000} & 0.24 \\
\hline & Has play ground & $72(36.7 \%)$ & $54(50.5 \%)$ & $18(20.2 \%)$ & & & & $(0.131,0.472)$ \\
\hline \multicolumn{9}{|l|}{$\begin{array}{c}\text { Food } \\
\text { served }\end{array}$} \\
\hline \multirow{3}{*}{$\begin{array}{l}\text { Main } \\
\text { meal }\end{array}$} & Rice+beans & $64(32.7 \%)$ & $42(39.3 \%)$ & $22(24.7 \%)$ & \multirow[t]{3}{*}{2} & \multirow[t]{3}{*}{17.526} & \multirow[t]{3}{*}{0.000} & \\
\hline & Beans+ chapati & $94(48 \%)$ & $37(34.6 \%)$ & $57(64 \%)$ & & & & \\
\hline & Ugali+vegetables & $38(19.3 \%)$ & $28(26.2 \%)$ & $10(11.2 \%)$ & & & & \\
\hline \multirow{2}{*}{$\begin{array}{c}\text { Between } \\
\text { Break } \\
\text { meals }\end{array}$} & Tea + mahamri & $94(48 \%)$ & $82(76.6 \%)$ & $12(13.5 \%)$ & \multirow[t]{2}{*}{1} & \multirow[t]{2}{*}{13.287} & \multirow[t]{2}{*}{0.000} & \multirow{2}{*}{$\begin{array}{c}4.647 \\
(1.958, \\
11.025)\end{array}$} \\
\hline & Tea + cake & $102(52 \%)$ & $25(23.4 \%)$ & $77(86.5 \%)$ & & & & \\
\hline \multirow[t]{3}{*}{ Servings } & Once & $97(49.5 \%)$ & $94(87.9 \%)$ & $3(3.4 \%)$ & 2 & $139 . .734$ & 0.000 & \\
\hline & Twice & $77(39.3 \%)$ & $13(12.1 \%)$ & $64(71.9 \%)$ & & & & \\
\hline & $>$ twice & $22(11.2 \%)$ & $0(0)$ & $22(24.7 \%)$ & & & & \\
\hline Snacks & Chocolate & $56(28.5 \%)$ & $27(25.2 \%)$ & $29(32.6 \%)$ & 6 & 16.808 & 0.010 & \\
\hline & Chips & $43(22 \%)$ & $19(17.8 \%)$ & $24(27 \%)$ & & & & \\
\hline & Juice & $36(18.4 \%)$ & $27(25.2 \%)$ & $9(10.1 \%)$ & & & & \\
\hline & Biscuits & $14(7.1 \%)$ & $4(3.7 \%)$ & $10(11.2 \%)$ & & & & \\
\hline & Crisps & $13(6.6 \%)$ & $6(5.6 \%)$ & $7(7.9 \%)$ & & & & \\
\hline & Ice cream & $25(12.8 \%)$ & $17(15.9 \%)$ & $8(9 \%)$ & & & & \\
\hline & Sweets & $9(4.6 \%)$ & $7(6.5 \%)$ & $2(2.2 \%)$ & & & & \\
\hline
\end{tabular}

\section{DISCUSSION}

The study findings show that from 196 respondents who took part, $51.5 \%$ were male and $48.6 \%$ were from public schools. Most of the respondents received a daily pocket money of between Ksh. 100-200 (54.6\%) and about $60.7 \%$ were from private schools. The common means of transport was school van $(68.9 \%)$, where $78.9 \%$ used it in private schools. About $77.6 \%$ of the respondents could spend their leisure time watching video games, where $91.2 \%$ from private schools and majority of the respondents $(33.7 \%)$ were in grade four. Study findings from Lilian (2010) show $31 \%$ of the children spend their leisure time watching television, playing (30\%) and

$38.2 \%$ of the respondents used school bus to school. While according to O'Loughin (2001), Multivariate studies have found that television viewing and playing video games for longer periods of time promotes obesity
The socio demographic factors of their parents or guardians show that $95.5 \%$ of private school parents were living in their personal houses compared to $57 \%$ of those in public schools. Majority (96.6\%) parents with children in private schools were employed and get a monthly income of over ksh. 20,000 (93.3\%) compared to $55.1 \%$ and $64.5 \%$ consecutively for those in public schools. About $80.6 \%$ of the parents were married

Based on school environment; study found out that $67.4 \%$ of the private schools had sport equipments. However, public school participated most in sport activities $(33.6 \%)$ and were having sport lessons regularly $(39.3 \%)$. About $79.8 \%$ of private schools were located within residential areas with only $20.2 \%$ having a play ground for children. Similar study by Lilian (2010) show that $98.3 \%$ of children have a positive attitude towards physical activity being enjoyment of the activities they indulged in during the 
lessons while others it gave a break to that monotony of being in class for long hours.

Up to $43.9 \%$ of the respondents were served food in school, $48.3 \%$ of the respondents in private schools could still receive food from outside school. The common main food served were rice with chapati $(64 \%)$ in private school and rice with beans $(32.7 \%)$ in public school, tea with cake $(86.5 \%)$ in private schools and tea with mahamri (76.6\%) in public school consecutively during tea break. The number of servings being once $(87.9 \%)$ in public schools and twice $(71.9 \%)$ in private schools. The common snack by majority of the respondents was chocolate $(28.5 \%)$. Other studies show increased intake of sugary diet is a risk factor to obesity by $66 \%$ (Wilson, 2000), chocolate is a common (62\%) snack in school going children very rich in fats consumed by children (Lilian, 2010) and persons with frequent snack intake are prone to obesity (Jahnsl, Siega and Popkin, 2001). Food sourced from outside school school by hawkers or vendors do not meet requirement of nutrition and health (Westanhoefer, 2001). Different study findings by Gibson (2000) show pre-school children to be more prone to obesity than school going due to carbohydrate intake.

The children anthropometric measurements of the respondents found out that there were $2 \%$ underweight (one in private and one in public school), $69.1 \%$ had a normal BMI in public schools, $28.1 \%$ were found to be over weight in private schools, $32.6 \%$ were obese in private schools.

Based on bivariate analysis, the following were found to be statistical significant; physical activities $(\mathrm{p}=0.000)$, eating habits $(\mathrm{p}=0.000)$, school location $(\mathrm{p}=0.000)$, food served either main or tea break meals $(\mathrm{p}=0.000)$ and snacks eaten by children $(\mathrm{p}=0.010)$. While the logistic regression analysis found out residence with AOR of 29.46, $(10.054,86.34)$ and food served between break with AOR of 4.647, (1.958, 11.025)

\section{CONCLUSION}

The proportion of obesity in lower primary school children stands at $23 \%$. The private school children being more vulnerable with $32 \%$ obesity and overweight $28.1 \%$

The school learning environment contributes majorly to the reported cases of obesity in children. This was visible by looking at physical activities children undertake in school, eating habits, school location, food and snacks children get in school

The parent's occupation, level of income, how children spend their leisure time at home as well means of transport to school being a contributor to childhood obesity.

\section{RECOMMENDATIONS}

The study recommends on the following;

1. The ministry of education to embark on sensitization of schools and parents the importance of child in school and home in order to reduce chances of lifestyle diseases

2. The government should be strict on the items required to be put in place by existing and upcoming schools in order to ensure learning environment is conducive to children

3. The parents should play their role to ensure children always take appropriate diet in their meals and assist children choose the best leisure activity for themselves

\section{REFERENCES}

1. Bertone E.R, Staneck E.s, \& Cohen N.L. (2003). Association between eating patterns and obesity in a free living US adult population. American Journal of Epidemiology, 158:85-92

2. Caroli M, Burniat W. (2002). Dietary management In: Childhood and adolescent obesity causes consenquences, prevention and management. Cambridge University Press, pp, 282-306

3. Cochran, J.J. (2010). Statistics Without Borders Assists with Haitian Data Collection Project. Amstat News, 8 (395), 18-19.

4. Jahhns L, Siega-Riz A.M. \& Popkin B.M. (2001). The increasing prevalence of snacking among USchildren .Journal of Pediatrics: 138:493-498

5. Kamau W. Jane (2008). Prevalence, intervention and management of overweight and obesity among primary school children in Nairobi Province. Post graduate thesis, Kenyatta University

6. Lilian Adhiambo Aballa (2010). Prevalence and risk factors for obesity among school aged children in Nairobi Province, Kenya. Master thesis, Kenyatta University

7. Malla J. (2004). Obesity and factors that contribute to obesity among Pre-adolescecnt attending $d$ ay private primary schools in Nairobi. Masters thesis, Kenyatta University

8. Ministry of Education Science and Technology (MOEST) (2018). Registered schools in Mombasa County. (Department of Statistics) Nairobi, Kenya

9. Mugenda O.M. and Mugenda A.G.. (1999). Research Methods. Quantitative and Qualitative approaches, ACT press Nairobi Kenya

10. O'loughlin, J. 7 \& Gray -Donald, K. (2000). One and two year predictors of excess weight gain among elementary school children in multiethnic, low-income. Inner-city neighborhoods. American Journal of Epidemiology; 152:739-746 
11. Westonnoefer J. (2001). Establishing good dietary habits in capturing the minds of children, Public Health Nutrition; 4:125-129

12. Wilson J.F. (2000). Lunch eating behavior of preschool children: effects of age, gender and type of beverage served. Physiological Behavoir; 70:27-33 